\title{
Nurse-led telephone review service for mild inherited bleeding disorders improves attendance rates, frees hospital resources and is highly rated by patients
}

Sharon Alavian, Wendy Hutchinson, Abigail Morris, Heather Williams, Debra Pollard

Introduction and objective: In the UK, the National Service Specification for haemophilia stipulates that all patients with mild inherited bleeding disorders must be reviewed annually by their haemophilia centre. For those patients who rarely experience problems relating to their bleeding disorder, attending a yearly hospital-based appointment may be viewed as a low priority. This can result in missed appointments and disconnection from their haemophilia centre,

\section{SHARON ALAVIAN}

Clinical Nurse Specialist, Hammersmith Hospital

Haemophilia Centre, Imperial Healthcare NHS Trust,

London, UK. E-mail: sharon.alavian@nhs.net

WENDY HUTCHINSON

Clinical Nurse Specialist, Hammersmith Hospital

Haemophilia Centre, Imperial Healthcare NHS Trust,

London, UK. E-mail: wendy.hutchinsonanhs.net.uk

ABIGAIL MORRIS

Clinical Nurse Specialist, Haematology Day Unit, The Royal

London Hospital Haemophilia Centre, Barts Health NHS

Trust, London, UK. E-mail: abigail.morris1@nhs.net

\section{HEATHER WILLIAMS}

Haemophilia Operational Services Manager,

Haematology Day Unit, The Royal London Hospital

Haemophilia Centre, Barts Health NHS Trust, London, UK.

E-mail: heather.williams10@anhs.net

DEBRA POLLARD

Lead Haemophilia Nurse, Katharine Dormandy Haemophilia \& Thrombosis Centre, Royal Free NHS Foundation Trust.

London, UK. E-mail: debra.pollardenhs.net

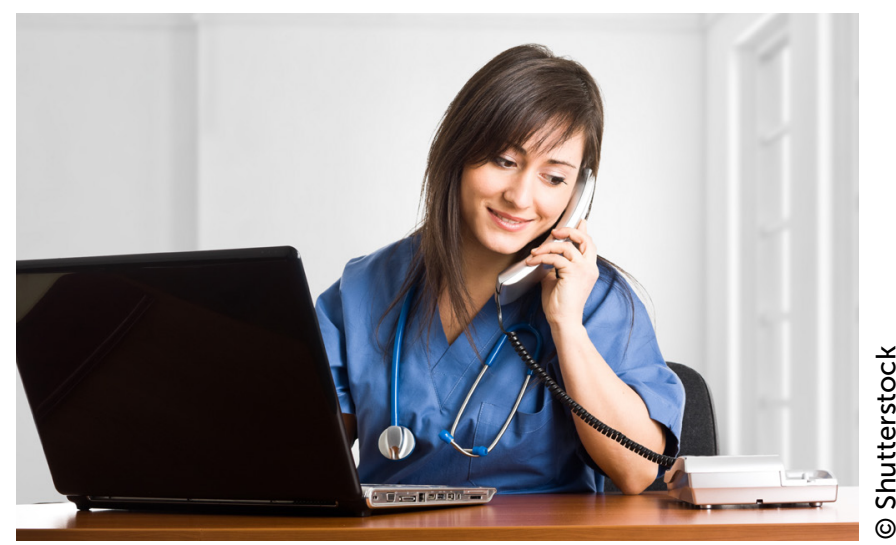

Nurse-led telephone clinics could provide a viable alternative to traditional hospital-based appointments for annual reviews of patients with mild inherited bleeding disorders

leading to poor understanding of how to manage their condition in emergencies, or when surgical or other invasive procedures may be necessary. The inherited nature of these conditions also has implications for reproduction, and it is of vital importance that the risk of bleeding around labour, delivery and the neonatal period are fully understood and mitigated against. The introduction of a structured, nurse-led telephone clinics across the North London Adult Haemophilia Network (NLAHN) offered an alternative method for patients to be reviewed. This strategy was then evaluated to assess whether the needs of patients were being fulfilled. Materials and methods: Clinical nurse specialists (CNS) from the NLAHN devised a short service evaluation questionnaire with Likert scales and

This is an Open Access article distributed under the terms of the Creative Commons Attribution-NonCommercial-NoDerivs License (https://creativecommons.org/licenses/by-nc-nd/3.0/) which permits use and distribution in any medium, provided the original work is properly cited, the use is non-commercial, and no modifications or adaptations are made. Copyright is retained by the authors. 
one open question. Patients across the NLAHN sites who had received a telephone review in 2016 were sent an anonymised questionnaire, with a stamped addressed envelope and a six-week return date. Results: 514 questionnaires were distributed, 174 were returned, and 18 were excluded as returned incomplete, giving a return rate of $28 \%$. Overall, $89 \%(139 / 156)$ of patients rated the new service between excellent and very good; $89 \%(139 / 156)$ reported that they were very satisfied with the information received in the review; and $95 \%$ (149/156) were happy to continue to receive telephone reviews. Conclusion: Patients found the telephone reviews a viable alternative to traditional hospitalbased appointments. The telephone clinics are more convenient for patients in terms of time and resources; they also helped those surveyed to re-engage with their haemophilia centre, ensuring continued education about their condition and the services offered. Overall attendance rates for the follow-up of patients with mild bleeding disorders have improved, with a reduction in traditional clinic appointments for this group. This has an ongoing positive impact on waiting lists and the financial burden of missed hospital appointments without impacting patient care.

Keywords: Haemophilia, Mild inherited bleeding disorders, Nurse-led, Review, Telephone clinics

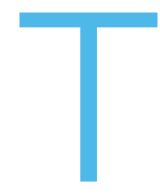

he term 'inherited bleeding disorder' (IBD) refers to a number of genetically inherited disorders affecting clotting function.

These incorporate rare but more commonly known disorders such as haemophilia A, haemophilia $B$ and von Willebrand's disease, as well as Factor I, II, $\mathrm{V}, \mathrm{VII}, \mathrm{X}, \mathrm{XI}$ and XIII deficiency, and disorders of platelet function ${ }^{[1]}$. The presentations and phenotypes of these conditions range from mild through to severe, depending on the type or level of deficiency.

For those people with mild IBDs who tend to only bleed following surgery, invasive procedures, injury, or a haemostatic challenge such as pregnancy, a yearly clinical review is sufficient to enable patients to receive updated information, ongoing support and education about their condition. Having an annual review also fulfils the requirements set by healthcare commissioners in the UK National Service Specifications (NSS) for bleeding disorders, which require patients be reviewed yearly in order to ensure continual registration at their haemophilia treatment centre (HTC) ${ }^{[2]}$.

Under the current UK NSS criteria, annual reviews for people with IBDs must include a documented two- way conversion between the healthcare provider and the patient. Within the North London Adult Haemophilia Network (NLAHN), this includes:

- A review of bleeding symptoms since last review;

- A review of current medications and any new health issues arising;

- Questions relating to dentistry and oral health;

- Discussion of any planned medical/surgical or dental procedures that may require a treatment plan in view of the bleeding disorder;

- Allocated time to discuss any concerns relating to the patient's bleeding disorder, including clarification, education and updates where appropriate, and any family issues arising as a result of the patient's bleeding disorder.

\section{Hæmophilia ح-London \\ The North London Adult Haemophilia Network}

NLAHN is comprised of two comprehensive care centres and one haemophilia centre: The Royal Free Hospital, The Royal London Hospital and Hammersmith Hospital. At the time of writing, there are more than 2000 patients registered within the network who have a mild IBD.

Although the London region is well served with transport links, travel times for patients to attend their haemophilia treatment centres can be excessive: some record journeys of up to three hours each way for an appointment that may last 15-20 minutes. Even for those with shorter journeys, at least a half a day may be lost from work or education/training. This may be burdensome for people with a mild IBD who have rarely or never experienced a bleed that required medical attention, especially if they feel they have no current medical issues to discuss. As reviews are essential for haemophilia centres to stay up to date with the patients, a viable alternative was required. A nurse-led telephone consultation was identified as one such method.

It was proposed that people with a mild IBD were offered a structured nurse-led telephone clinic with their clinical nurse specialists (CNS) for two consecutive years, with a traditional hospital review every three years. Adopting this type of review was perceived to be potentially beneficial, as it met the healthcare commissioners' requirement for a yearly review for those with a mild IBD, while being potentially more convenient for patients, and also a better use of the time and resources available within HTCs. 


\section{PURPOSE/OBJECTIVES}

The purpose of the nurse-led telephone clinics was multifaceted, taking into account patient care, effective use of resources and the HTC's obligations under the NSS. Working together, CNSs from across the NLAHN agreed a process for conducting nurseled telephone review clinics and consensus on which patients were eligible to be referred to this service. An Advanced Clinical Practice Guideline and Standard Operating Procedure (SOP) were prepared for the clinical and administrative teams, agreeing the roles and responsibilities required to undertake the clinic with standardised templates.

The reviews themselves were designed to:

- Disseminate up-to-date information

- Provide education on identification, early intervention and management of bleeds

- Re-engage with patients who may not have attended for a review for some time

- Reduce the 'did not attend' (DNA) rate

- Offer a viable alternative method for patients to be reviewed

- Increase cost-effectiveness

- Fulfil the health commissioning requirement for registration at HTCS.

Any patient that fulfilled the inclusion criteria was eligible to receive a review via the telephone clinic. The criteria for inclusion required patients to be 18 years of age or over with a mild IBD, without severe arthropathy or other significant comorbidities, reviewed by their HTC within the previous three years, and with a good command of spoken English. It was also required that patients had no issues that could make it difficult to communicate via telephone and were not assessed as being a vulnerable adult. Inclusion and exclusion criteria are shown in Figure 1.

While continuing to offer up-to-date information and education on their care and management, engaging patients with mild IBDs in a telephone review would ensure ongoing support and contact with their healthcare professionals and also eliminate travel times. The NLAHN haemophilia CNS group believed that the convenience to patients of only needing to attend every third year for a hospital consultation could help to reduce the number of missed appointments, and thereby increase the availability of clinic slots for patients that require them.

Across the NHS, one in 10 hospital appointments are missed every year ${ }^{[3]}$. Cost savings could be realised by actively trying to reduce DNA rates. Research has shown that simple measures such as sending a text message five days prior to a patient appointment, with the date, time, specific financial cost to the NHS for not attending and including contact details to use to cancel/rearrange their appointment, reduced missed appointments by a relative reduction of $23 \%{ }^{[3]}$.

Following the implementation of the telephone review service, the NLAHN CNSs proposed to seek patient input as a means of evaluating its effectiveness.

\section{METHODS}

A survey was created to assess patients' experience of the telephone clinic service and to seek their opinions and ideas for its further development. Approval for the survey was obtained from the NLAHN Board.

All patients with a mild IBD that had participated in a nurse-led telephone clinic review in the year 2016 received a copy of the survey to complete. The survey comprised an anonymised short survey questionnaire using a Likert scale, with one open question to allow

Figure 1: Inclusion and exclusion criteria for review via telephone clinic

\begin{tabular}{|c|c|}
\hline INCLUSION CRITERIA: & EXCLUSION CRITERIA: \\
\hline $\begin{array}{l}\text { - Patients } 18 \text { years of age and over } \\
\text { - Mild inherited bleeding disorder } \\
\text { - Patients without severe arthropathy or other significant } \\
\text { comorbidities } \\
\text { - Good command of spoken English } \\
\text { - No issues that may make it difficult to communicate via } \\
\text { the phone } \\
\text { - Not assessed as a vulnerable adult according to National } \\
\text { Health Service (NHS) guidance (e.g. mental health or } \\
\text { mental capacity issues) }\end{array}$ & $\begin{array}{l}\text { - New patients } \\
\text { - Assessed by the consultant as not suitable for nurse-led } \\
\text { telephone reviews } \\
\text { - Patients with a preference to attend the HTC for a review } \\
\text { - Patients where the last two consecutive attempts to } \\
\text { contact them for booked appointments has been } \\
\text { unsuccessful. }\end{array}$ \\
\hline
\end{tabular}


free feedback (see Figure 2). A stamped and addressed envelope was included, with a six-week return date to the patient's HTC.

Once the completed surveys were returned, the results were collated and analysed by the authors, and subsequently shared by CNSs in the NLAHN hospitals as a service evaluation. The results have also been shared more widely in a presentation at the World Federation of Hemophilia Congress Nurses' Workshop in 2018.

\section{RESULTS}

Across the NLAHN, 583 people with IBDs were booked into the telephone review clinic in 2016. Of these, $88 \%$ (514/583) completed their reviews; 80\% (472/583) engaged with their initial telephone review clinic. Of those that did not attend their initial appointment, $39 \%$ (45/114) engaged with a subsequent telephone appointment. Only 12\% (69/583) of all patients that were offered a telephone review in 2016 have not had one - these patients were excluded from the survey.

A total of 558 questionnaires were distributed. 174 were returned, of which 18 were excluded as returned incomplete, giving a return rate of $28 \%$.

Overall, 99\% (154/156) of people were aware that they were booked into a nurse-led telephone review clinic. All patients who are scheduled for the telephone clinic should receive information about this nurse-led service and have the choice to opt out and remain in traditional hospital-based follow-up. This result demonstrates that this communication has been successful.

Those patients who were aware that they were having a telephone clinic review were asked if they were contacted at the correct time and date. The majority $(87 \% ; 134 / 154)$ responded that they were very satisfied the nurse had contacted them at the correct pre-arranged date and time; $7 \%(11 / 115)$ were somewhat satisfied; $3 \%$ (5/114) were neither satisfied nor dissatisfied; 3\% (4/114) reported being somewhat dissatisfied. No one reported being very dissatisfied.

When patients were asked to rate how convenient they found the telephone review in comparison with a standard hospital appointment, the majority (93\%; 145/156) reported that they had found it either extremely convenient or very convenient; $6 \%(9 / 156)$ that they found it somewhat convenient; and $1 \%(2 / 156)$ not so convenient.

In terms of overall satisfaction with the information received during the telephone clinic, 89\% (139/156) of respondents reported being very satisfied; 6\% (9/156) somewhat satisfied; $1 \%$ (2/156) somewhat dissatisfied; and 4\% (6/156) neither satisfied nor dissatisfied. No respondents reported being very dissatisfied with the information given to them.

The amount of time that patients require for their telephone review inevitably varies depending on their individual needs. With regard to this aspect of the telephone review, 95\% (148/156) reported that they were either very satisfied or somewhat satisfied with the amount of time given; 5\% (8/156) were neither satisfied nor dissatisfied.

Patients were asked if they were satisfied that they were given the opportunity to ask questions during their telephone review, with 94\% (146/156) rating this between excellent or very good; $5 \%(8 / 156)$ reporting it as good; and $1 \%(2 / 156)$ reporting it as fair. No respondents reported this as being poor.

In terms of the answered provided to the questions asked by patients, 95\% (148/156) were very satisfied or

Q1: Were you aware of your telephone clinic review appointment? [YES / NO]

Q2: If you were aware, are you satisfied that you were called at the date and time agreed?

Q3: How convenient, compared to the standard hospital appointment, was your telephone review?

Q4: Are you satisfied that you received adequate information / education regarding your haemophilia / bleeding disorder?

Q5: How satisfied were you with the amount of time given to you during the telephone consultation?

Q6: How do you rate the opportunity given to you to ask questions?

Q7: How satisfied were you with the answers provided to your questions?

Q8: Overall, how did you find the telephone consultation as an alternative to a face-to-face appointment?

Q9: How would you rate the telephone review service?

Q10: In future, would you be happy to have further telephone reviews? [YES / NO / NOT SURE]

Figure 2: Questions asked in the NLAHN telephone review service evaluation. Unless indicated, the questions used a five-point Likert scale, with 5 as the greatest level of satisfaction and 1 the least 
Q2: Satisfaction with contact at correct date and time

Q3: Convenience of telephone review vs. hospital-based review

Q4: Satisfaction with information/education received

Q5: Satisfaction with time given to telephone review

Q6: Satisfaction with opportunity to ask questions

Q7: Satisfaction with answers provided to questions

Q8: View of telephone review as an alternative to face-to-face review

Q9: Overall rating of telephone review service
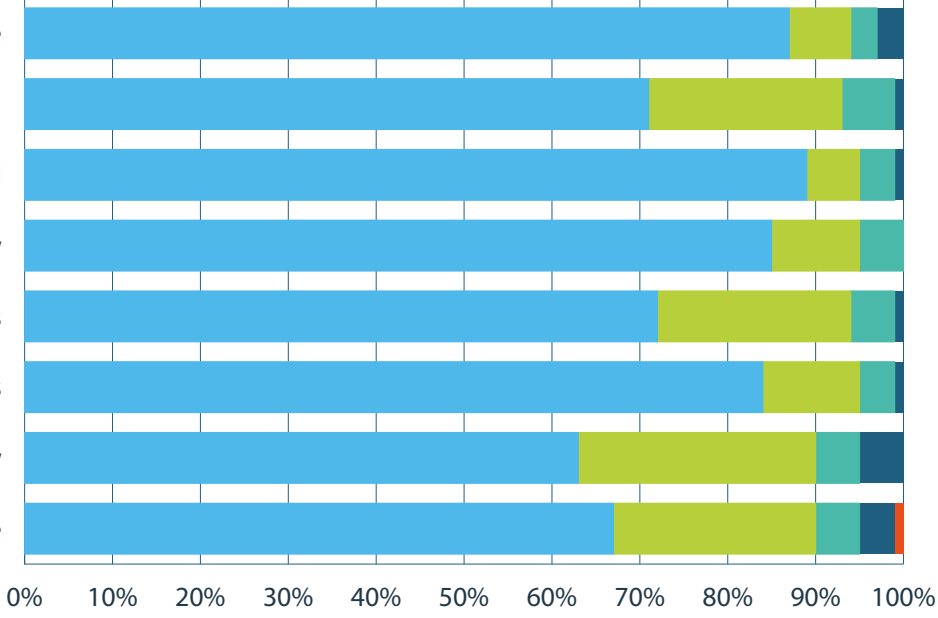

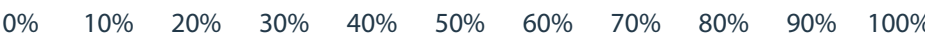

5: Very satisfied / Excellent

3: Neither satisfied nor dissatisfied / Good

4: Somewhat satisfied / Very good

2: Somewhat dissatisfied / Fair

1:Very dissatisfied / Poor

Figure 3: Patient responses to questions 2 to 9 of the NLAHN telephone review evaluation, using a five-point Likert scale, where 5 denotes the highest level of satisfaction and 1 denotes the lowest level of satisfaction

somewhat satisfied with the answers; $4 \%(7 / 156)$ did not have a preference either way. One respondent reported being somewhat dissatisfied; no respondents reported being very dissatisfied with the answers given by the CNS to their questions.

The majority of patients $(95 \% ; 148 / 156)$ reported that the telephone consultation as an alternative to face-to-face consultation was either excellent or very good; $5 \%(8 / 156)$ viewed the telephone consultation as fair, compared to the face-to-face consultation. No respondents rated the telephone review as a poor alternative to face-toface consultation.

The majority of respondents $(95 \% ; 148 / 156)$ rated the telephone review service between excellent and good, with only one person saying it was poor, and a further $4 \%(7 / 156)$ people rating it as fair.

When asked if they would be happy to continue having telephone clinic reviews, 95\% (149/156) said that they would; $4 \%(6 / 156)$ said they would not be happy; and one person was unsure.

\section{DISCUSSION}

\section{Timing of appointments and convenience of telephone clinics}

As the CNSs organise their own workload and know their patient group well, they were able to predict with some accuracy the individual patients that may require a slightly longer time slot for their telephone review. Some centres were able to account for and accommodate longer time slots when the appointments were booked, but in others this was not possible. This may be reflected in the $6 \%$ of people who reported that they were not called at the correct date and time.

Although 94\% (145/154) of respondents were happy that they were contacted at the correct time and date, the aim is, of course, $100 \%$. One person commented:

\section{"[My] only slight complaint is having to wait by the phone when the call is delayed for a length of time. I realise this is not always under the control of the nurse."}

CNSs do try to contact patients at the allocated time agreed; however, occasionally this is not possible. If the CNS is delayed due to unforeseen circumstances, the standard should be that, at the earliest possible time of knowing that there will be a delay, either the CNS or another member of the team contacts the patient to apologise and re-arrange the appointment.

All patient contact details need to be kept up to date and the CNSs also need to ensure that they have confirmed the preferred telephone number on which to contact the patient. One person commented:

\section{"Calling my home phone during office hours is just ridiculous."}

The same respondent also iterated that while people are increasingly reliant on mobile phones, 
some people live or work in places with poor mobile telephone reception. It may therefore be necessary for patients to provide an alternative landline number for the CNS to contact them on.

Most respondents remarked on how convenient the telephone clinic, saving them both time and money and providing more flexibility. Comments included:

\section{"Excellent and much more convenient."}

"It is very convenient to have these telephone reviews as I get the same thing out of it las a hospital-based review] and it saves me a long trip into London with a little one/or finding childcare."

"I think it is a good idea. It saves me travelling to the hospital for what is, for me, a relatively short appointment."

"I live a long way from my haemophilia centre - telephone appointments save at least half a day of my time and around $£ 50$ in costs. Much appreciated!"

"Very convenient if you have a full-time job."

However, it must be acknowledged that this type of review may not suit or be appropriate for everyone: a select few will always prefer to attend in person and their preference needs to be respected.

Patients commented that:

"I like face-to-face, nothing else can trust what's going on. I need to speak face to face."

"The telephone service was good; however I do prefer to see someone for a face-to-face appointment. As my daughter has a yearly appointment I usually come for my appointment at the same time."

"...actually I do miss my visits and seeing the staff friends I have made over the many years."

The timing of the clinic review was also commented on, with one patient saying:

"I would be grateful if the time slot for telephone consultation could be more suitable for working and school attendees, [such] as lunch time or after school times."
Clinics, including the telephone review clinic, are generally scheduled for either morning or afternoon sessions, and the HTCs will try to accommodate most requests at the time of booking the appointment where possible. This helps to ensure that having a telephone review does not become an extra burden for time-pressured people.

While the telephone clinic fulfils the NSS criteria for a review, as well as potentially helping some people to re-engage regular contact with their centre, patients are fully aware that they can contact or attend their HTC at any time and can choose to attend a face-toface consultation.

\section{Patient satisfaction with the telephone review} Overall, patients were positive about their experience of the telephone review clinic.

As with a face-to-face consultations, there is an allocated time slot for telephone reviews. These vary in length between hospitals, ranging from 20-30 minutes, but fluidity is employed depending on the individual needs of the patient. By comparison, the allocated face-to-face time slots for hospital-based follow-up reviews ranges from 15-20 minutes.

Long waiting times in outpatient departments has been a persistent criticism since the inception of the NHS in $1948^{[4]}$. Patients are frequently not seen on time when attending hospital-based clinic appointments due to the unavoidable overrunning of the clinics, meaning that patients can sometimes feel rushed ${ }^{[5]}$. However, no respondents reported being somewhat dissatisfied or very dissatisfied with the length of time of their telephone review, and comments indicated that they felt they had more time, with one patient saying the review was "well structured":

\section{"Both acceptable, but phone consultation gives time."}

"I feel that the time given for the telephone review is exactly what I would be given during a face-to-face. The clinical staff are excellent."

Comments from the patients regarding quality of information were very positive and included:

"The quality has been very high so far."

"The staff and clinical care proved by the clinic is world class. This is a great example of how the NHS could work better." 
"... staff who have been there for years are very efficient and knowledgeable."

"The nurse I spoke to was always very helpful with my bleeding disorder..."

"The staff are brilliant. They're fantastic." This very positive feedback from the patients may, in part, reflect the fact that all the nurses involved in the telephone clinic are CNSs who have advanced practitioner skills, completed a specific competence assessment tool prior to starting the clinic, and are able to disseminate up-to-date information.

For patients who may be hard of hearing, this style of review may not always be appropriate; however, with a bit of thought and consideration, they may also benefit. One patient commented:

\begin{abstract}
"I am hard of hearing and the Nurse Specialist was very sensitive to this and spoke slowly and clearly, for which I was most grateful. The telephone call went well and I felt that the issues discussed were clearly covered certainly to my satisfaction."
\end{abstract}

Although respondents were satisfied overall with the information they received, $1 \%$ reported being somewhat dissatisfied and commented on their need to have a yearly blood test in order to check their factor levels:

\section{"I very much regret that I can't see the usefulness of telephone appointments. Although the staff I have spoken to have invariably been kind and their questions comprehensive, I fail to see how any judgement can be made about a blood condition without conducting a blood test to measure the current factor level."}

This comment indicates a belief that factor levels may change significantly from year to year. Although the patient reported being happy with the information they had received in the telephone clinic review, the comment highlights the need for CNSs to clarify and explain factor level results to patients.

Patient satisfaction with the opportunity to ask questions during their telephone review and the answers they received not only indicates that the CNSs conducting the telephone clinics had the right level of knowledge and expertise, but may also reflect the quality of the relationships between the CNSs and their patients. All of the NLAHN CNSs have well established relationships with their patient group, through which they have built up a mutual feeling of trust and respect. This enables patients to feel confident and at ease to ask any questions they may have. Patients commented that:

"Staff are excellent and always allow ample time, ensuring there are no issues left to discuss or concerns you may have."

"Your staff are most helpful."

"I feel that the Haemophilia Team at the Hospital are genuinely patient-centric. They have the time, compassion, competence and resources to ensure that all my questions are answered and my needs met."

Although the results were good in this regard, CNSs nevertheless need to ensure that they ask patients if they have any further questions at the end of every consultation. This helps to ensure that all patients feel they have been listened to, understood and had their issues addressed.

\section{Telephone vs. hospital-based review}

Generally, respondents' comments on the telephone review service were very positive and complimentary;

"This is a 'good thing', please don't stop it."

"I am perfectly satisfied to continue with the telephone clinic service"

"It is a superb service, but I am quite a simple case."

"I believe it's an excellent service as an alternative to visiting the hospital just for usual reviews."

"Please continue - very convenient and fine for my condition. Much more preferable; please continue."

"Excellent idea and well handled. Saves time for patients and hospital alike."

However, while the majority were very positive about the telephone clinic being a viable alternative to face-to-face clinic, they wished to continue having a face-to-face appointment every two to three years. There was also a recognition that face-to-face 
consultations were preferable in the event of a problem. Patients commented:

"I did find the telephone appointment very good; however, it isn't the same as seeing someone face-to-face. As long as I get a chance to do that every so often, I am more than satisfied." "It works well. It is more efficient than attending in person. The offer of two telephone interviews and one physical is about right."

"I do like telephone clinics, but think it is good to have a face-to-face consult at least every other year."

"Would like to visit every two to three years."

"Face-to-face has a different quality and it is always better, but I found telephone review to be an excellent alternative when there are no major issues to address."

"Obviously if I were to have a problem I would prefer a face-to-face consultation, but for a normal check-up the telephone clinic is ideal."

In line with the agreed proposal set out with the commissioners, the CNSs would offer a telephone clinic for two consecutive years, at which point the third year's appointment would be a traditional face-to-face review. One of the aims of the telephone clinic was to help facilitate a more convenient way to encourage people to maintain regular contact with their centre. The feedback from patients indicates that having a telephone review helps them to stay engaged with their centres:

"I live a considerable distance, so for me this is an excellent service and maintains on going contact."

Only $5 \%$ (8/156) of respondents rated the telephone consultation as a "fair" alternative to face-to-face appointments, rather than good or excellent. One respondent felt that although they liked the option of a telephone clinic:

\section{"I fear some of the human side will be lost without} the annual appointment in person. Nevertheless, what you do and the way you do it when something medical arises, e.g. operations etc., is exemplary and is a credit to all."

The CNSs need to be mindful of this fear, as the human touch and being caring and compassionate are core values at the heart of nursing ${ }^{[6]}$, and should never be lost when dealing with patients, whether communicating face-to-face or over the telephone. It must also be remembered that while the majority may have indicated a preference for communicating by telephone, individual preferences should always be respected. Not everyone is comfortable asking questions over the phone, and as one respondent commented:

"I do prefer face-to-face appointments. I find it harder to talk [through] over the phone any queries I may have and tend to rush through the phone call."

\section{Looking forward}

With such positive confirmation from patients, it is clear that they valued this nurse led service. One respondent commented that it was:

"An excellent service. I would like for it to be extended to paediatrics too as my two children would benefit. Our condition is very mild so we don't need to be seen really. A catch-up on the phone would be totally sufficient, plus reduce the need to be taken out of school for hours."

While telephone reviews with a CNS are not currently offered to children or their parents, this is something that can be explored in the future. As the younger generation of people with bleeding disorders are very familiar with modern technology, different styles and types of reviews may soon be readily available which incorporate programs and apps including Skype, video calls, WhatsApp, FaceTime and Viber ${ }^{[7]}$. All of these options would facilitate the opportunity to talk with patients and also see them while conducting a review remotely.

As patients are central to this change in their reviews, they need to be actively encouraged to be involved in making informed decisions with regards to their care ${ }^{[7]}$, as well as participating in reviewing the telephone clinic on a regular basis through providing feedback.

Offering patients a viable alternative way to undertake a review without compromising their care has improved the convenience for patients. As patients are only required to attend hospital every third year, this model may help to reduce the current DNA rate for hospital appointments.

Figures suggest that increasing the number of patients accessing the nurse-led telephone clinics has the potential to substantially release more hospital face-to-face time slots for new patients and those with more complex health issues. 
Providing a nurse-led telephone clinic results in a cost saving as it is less labour-intensive for administrative/clerical staff and avoids both doctors and nurses waiting for patients that fail to arrive for their hospital appointments). Offering patients the option of having a telephone review clinic appointment has the potential to be a more efficient use of time and resources and may also help to re-engage some patients back into the service and reduce the overall DNA rates. It is therefore important that both doctors and nurses are proactive in ensuring that the momentum to identify and refer patients to the nurseled telephone service is maintained.

\section{CONCLUSION}

The result of this simple survey demonstrates that the nurse-led telephone review clinic offers a viable alternative from the traditional hospital face-to-face review and is well received by patients. It has fulfilled its initial aims, as not only does the service offer patients an alternative way to be reviewed, but it fulfils the criteria for a review. Patients find it is more convenient for them without compromising their care and management, and providing them with the opportunity to receive up-to-date information. Additionally, it complies with the requirement of the healthcare commissioners to deliver a yearly review to those with a mild IBD in order for them to be kept on the register.

Some patients that had previously failed to attend clinic appointments have now re-engaged with the service and are having telephone reviews with the CNS. Having a telephone review also means that less time slots for hospital-based clinics are wasted when patients fail to attend the clinic. This has led to an increase in valuable hospital time slots for patients with more complex health needs.

According to NHS Digital report for the year 20162017 , over $20 \%$ of hospital outpatient appointments in England were not attended ${ }^{[8]}$. To promote a good uptake for telephone clinic reviews, in addition to only offering a telephone review after patients have attended for a face-to-face consultation, it may be beneficial to send a text or telephone message as a reminder in advance of the appointment ${ }^{[9]}$. In the current electronic NHS system, it is not possible to send a telephone clinic reminder as the system is only programmed to send reminders to people attending the hospital. Discussions with the patient around receiving telephone clinic appointments need to be had during a face-to-face consultation to ensure that patient is well prepared and that the clinic has appropriate telephone numbers and an awareness of any other potential issues. To further improve the efficacy of telephone clinics, this option needs to be explored further.

The drive for advanced nurse practice in the UK has opened the door for CNSs to be more proactive in initiating alternative ways for the delivery of care ${ }^{[10]}$. Having the NLAHN CNS teams conducting the reviews has also allowed them the opportunity to extend their roles and utilise their own skills. Although some time slots have now been released for the hospital-based clinics, sufficient additional time needs to be allocated to CNSs to facilitate the telephone clinics, in order to ensure completion of all the necessary administrative tasks that clinics generate.

The telephone clinic has the potential to increase cost-effectiveness. Optimising the most efficient use of health professionals' time and resources is an area that still needs to be explored in terms of helping to reduce overall costs. However, the potential cost savings of running a telephone clinic needs to be offset against the time and resources required for CNSs and administrative teams to facilitate the clinic. Further review will be necessary to accurately determine the financial impact that these clinics have had in the NLAHN centres.

To further enhance communication with patients through modern technology, we also need to explore the possibilities beyond the use of conventional telephones for reviews ${ }^{[11]}$. Smart phones, iPhones/iPads, tablets and computers offer the possibility of using Skype, video calls, WhatsApp, FaceTime and Viber, for example, which would enable CNSs to see the patients as well as communicate with them in real time. This has the potential to truly revolutionise the way that reviews are conducted in the future, especially in remote communities.

\section{RECOMMENDATIONS}

As the nurse-led telephone clinic has received overwhelmingly positive responses from patients in the NLAHN area, the impetus to identify and refer patients to the this must continue.

After a patient has attended a clinic review in hospital, doctors and nurses should be proactive in referring appropriate patients to the telephone clinic for their next appointment. Informing patients of the telephone clinic in the NLAHN patient newsletter may also help in engaging patients with the new service.

To help optimise a higher return in survey response rates, exploring the possibility of using online computer tools, such as Survey Monkey, to capture patient feedback, instead of posting out paper surveys, may also help. 
In order to further facilitate new ways of conducting reviews, there is a real need to explore the possibilities that modern technology can offer. This could transform the way that clinics are conducted in the future.

Utilising the skills of the CNS is an avenue that should be explored further. Having specialist nurses reviewing people with mild IBD via telephone clinics could free up some hospital time slots for patients with more complex issues. It should be borne in mind, however, that patients with mild IBDs are sometimes less knowledgeable and less able to cope with relatively straightforward issues relating to their condition compared to those with severe IBDs, and that there will inevitably be occasions when it is better for those with mild IBDs to attend hospital in person.

Finally, to further explore, improve and develop the NLAHN telephone clinic model, it would be beneficial to run pilots through other local and/or regional haemophilia networks and treatment centres.

\section{ACKNOWLEDGEMENTS}

The authors have advised no interests that might be perceived as posing a conflict or bias.

This article reports on a survey to which participants responded knowing any comments may be reported.

\section{REFERENCES}

1. White GC, Rosendaal F, Aledort LM, et al. (2001) Definitions in haemophilia. Recommendation of the scientific subcommittee on factor VIII and factor IX of the scientific and standardization committee of the International Society on Thrombosis and Haemostasis. Thromb Haemost 2001; 85(3): 560.

2. The Haemophilia Alliance. A National Service Specification for Haemophilia and Related Conditions. London:
The Haemophilia Alliance, 2006. Available at: http://www. ukhcdo.org/docs/HaemAlliance-NatSvsSpec2006.pdf (accessed 29 June 2018).

3. Department of Health $\&$ Social Care. A zero cost way to reduce hospital appointments. Research and analysis. 4 January 2016. Available at: https://www.gov.uk/government/ publications/reducing-missed-hospital-appointments-usingtext-messages/a-zero-cost-way-to-reduce-missed-hospitalappointments (accessed 29 June 2018).

4. Welch JD, Bailey NTJ. Appointment systems in hospital outpatient departments. Lancet 1952; 259(6718): 1105-8.

5. Dugdale DC, Epstein R, Pantilat SZ. Time and the patientphysician relationship. J Gen Intern Med 1999; 14(suppl 1): S34-40.

6. NHS Five Year Forward View. October 2014. Available at: https://www.england.nhs.uk/wp-content/ uploads/2014/10/5yfv-web.pdf (accessed 29 June 2018).

7. Khair K, Barker C, Bedford M, et al. A core competency framework for haemophilia nurses in the UK. The Journal of Haemophilia Practice 2014; 1: 32-6.

8. NHS Digital. Hospital outpatient activity, 2016-2017. 28 November 2017. Available at: https://digital.nhs.uk/data-andinformation/publications/statistical/hospital-outpatient-activity/ hospital-outpatient-activity-2016-17 (accessed 8 August 2018).

9. Gurol-Urganci I, de Jongh T, Vodopivec-Jamsek V, Atun R, Car J. Mobile phone messaging reminders for attendance at healthcare appointments. Cochrane Database Syst Rev 2013;12: CD007458.

10. Department of Health. Advanced level nursing: a position statement. London: Department of Health, 2010. Available at: https://www.gov.uk/government/uploads/system/uploads/ attachment_data/file/215935/dh_121738.pdf (accessed 29 June 2018)

11. Department of Health \& Social Care. Personalised health and care 2020: a framework for action. Policy paper. 13 November 2014. Available at: https://www.gov.uk/government/ publications/personalised-health-and-care-2020/using-dataand-technology-to-transform-outcomes-for-patients-andcitizens (accessed 29 June 2018).

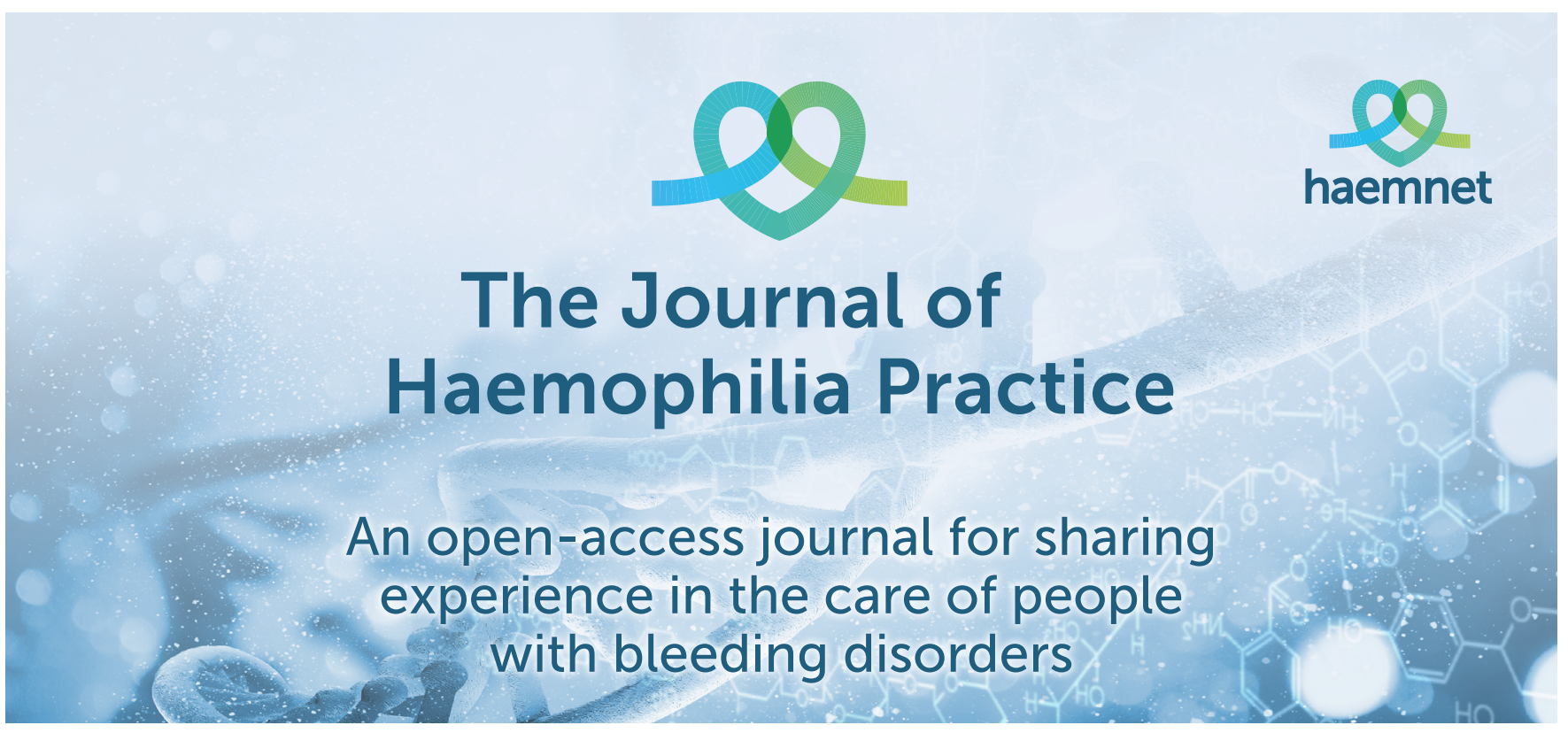

